\title{
Aprendizaje autónomo del estudiante apoyado en recursos audiovisuales en el contexto de un Grado de Ingeniería Informática: experiencias con metodologías de enseñanza activas ${ }^{*}$
}

\author{
Enrique Vallejo ${ }^{1}$, Pablo Fuentes ${ }^{1}$ y Mariano Benito M $^{2,1}$ \\ ${ }^{1}$ Universidad de Cantabria, Santander, España \\ ${ }^{2}$ Recore Systems, Eindhoven, Holanda
}

\begin{abstract}
The course Introduction to Computer Networks from the University of Cantabria has a low success, with complaints about the lack of sufficient practical classes that reinforce the theoretical contents. An innovation project has been developed to develop a set of multimedia materials that replace some master classes and increase the amount of practical sessions, following a flipped classroom model. Specifically, six videos have been developed, each of them accompanied by a self-learning activity, and an associated practical session. Such sessions followed approximately a Problem-Based Learning approach.

The impact of the project has been evaluated, on the motivation, withdrawal and academic results. The response of the students is positive, with a slightly increase in the classroom attendance. Students indicate that they prefer this type of learning. However, monitoring tools show that activity attendance is significantly lower than expected. Academic results show a rate of student withdrawal similar to previous years, but a slightly higher rate of students passing the course. Overall, the results of the project is very positive, given the increased student motivation, and a similar approach will be considered in forthcoming courses.
\end{abstract}

Keywords: Multimedia learning resources, flipped classroom, self-learning.

\section{Resumen}

\footnotetext{
*Este proyecto está apoyado por la III Convocatoria de Proyectos de Innovación Docente del Vicerrectorado de Profesorado de la Universidad de Cantabria y por el Ministerio de Economía, Industria y Competitividad bajo el contrato TIN2016-76635-C2-2-R (AEI/FEDER, UE). Pablo Fuentes está financiado por el Ministerio de Educación, Cultura y Deporte, beca FPU13/00337. Trabajo realizado mientras Mariano Benito pertenecía a la Universidad de Cantabria.
} 
Aprendizaje autónomo del estudiante apoyado en recursos audiovisuales en el contexto de un Grado de Ingeniería Informática: experiencias con metodologías de enseñanza activas

\begin{abstract}
La asignatura Introducción a las Redes de Computadores de la Universidad de Cantabria presenta un rendimiento bajo, con queja de la falta de clases prácticas que refuercen los contenidos teóricos. Para atacar el problema, se ha desarrollado un conjunto de materiales audiovisuales que reemplacen algunas de las clases teóricas y aumenten la cantidad de sesiones prácticas en el aula siguiendo un modelo de flipped classroom. En concreto, se han desarrollado seis vídeos para el auto-aprendizaje por parte del alumno, acompañados de tests de autoevaluación y de seis sesiones prácticas en el aula. En dichas sesiones, se ha seguido aproximadamente una metodología de aprendizaje basado en problemas.

Se ha evaluado el impacto del proyecto sobre la motivación, seguimiento y resultados del alumnado. La respuesta del alumnado al cambio metodológico es positivo, aumentándose ligeramente la asistencia a clase y seguimiento de la asignatura. Sin embargo, el seguimiento de los recursos es mucho más bajo de lo esperado. Los resultados académicos muestran una tasa de alumnos presentados similar a la de cursos previos, pero una tasa de aprobados ligeramente superior. Globalmente, el resultado del proyecto es muy positivo, y plantea seguirse un esquema similar en cursos posteriores.
\end{abstract}

Keywords: Recursos docentes audiovisuales, clase invertida, autoaprendizaje.

\title{
1 Introducción
}

El exceso de clases magistrales está identificado como uno de los problemas más claros de cara a la motivación y seguimiento de los alumnos. El abuso de clases magistrales se justifica desde el profesorado con la necesidad de cubrir un cierto temario en un tiempo limitado, pero incide negativamente en la motivación del alumnado, el seguimiento de la asignatura y la adquisición de competencias, lo que finalmente afecta a los resultados académicos. En concreto, esto viene ocurriendo en la asignatura "G661 Introducción a las Redes de Computadores" del Grado en Ingeniería Informática de la Universidad de Cantabria. La asignatura presenta una tasa de aprobados baja, típicamente ligeramente inferior al $50 \%$. Una de las quejas recurrentes de los alumnos es la falta de suficientes clases de problemas en el aula que refuercen los contenidos teóricos estudiados en la asignatura.

Esta falta de más clases prácticas se asocia a la imposibilidad de cubrir el temario propuesto de la asignatura con más clases prácticas siguiendo la organización que se ha empleado en cursos anteriores. Diferentes metodologías propuestas para atacar este conjunto de problemas incluyen entre otras las clases invertidas (Lage y Treglia 2000; Santiago y Díez 2016), cambios de metodología como el uso de materiales audiovisuales (Ronchetti 2010), o estrategias de Aprendizaje Basado en Problemas (ABP) (Wood 2003). Estas propuestas han tenido mucho auge recientemente, motivadas por las plataformas de autoaprendizaje online como cursos MOOC basados en diferentes plataformas (Sparks 2011).

Para solventar la situación de la asignatura, en el curso académico 2016-17 se ha planteado un Proyecto de Innovación Docente. El objetivo de este proyecto es modificar la metodología docente de la asignatura, desarrollando para ello un conjunto de 
materiales audiovisuales que reemplacen algunas de las clases teóricas y que permitan aumentar la cantidad de clases de problemas en el aula. Los contenidos adquiridos en dichos materiales audiovisuales se refuerzan mediante pruebas de autoevaluación a través de la plataforma online de la asignatura, y se comentarán en la siguiente clase presencial.

Este cambio busca de forma directa un aumento del número de sesiones de prácticas en el aula. De forma indirecta, también se busca una mejora en los resultados de la asignatura por diferentes motivos: se obliga a los alumnos a llevar al día los contenidos y realizar una serie de actividades de autoevaluación; se espera mejorar la motivación del alumnado, gracias al trabajo continuo en la asignatura; y finalmente, se espera que esto se traduzca en un aprendizaje más profundo y una mejora en los resultados de la asignatura.

De esta forma, las contribuciones principales de este artículo son las siguientes:

- Se presenta un caso de aplicación de modificación de metodologías docentes en una asignatura de Ingeniería Informática, empleando materiales audiovisuales, clases invertidas y aprendizaje basado en problemas.

- Se presentan detalles técnicos del desarrollo de los contenidos, así como algunos problemas encontrados, principalmente problemas técnicos y aspectos que no resultan agradables para los alumnos como la duración de los vídeos.

- Se analizan los resultados del cambio metodológico aplicado, teniendo en cuenta la impresión informal de los alumnos, herramientas de monitorización de las actividades, encuestas de satisfacción y resultados académicos. La impresión global de los alumnos es muy positiva, si bien el seguimiento de las actividades es moderado. El seguimiento de la asignatura aumenta levemente, y con él la tasa de aprobados en la única convocatoria hasta la fecha.

\section{Realización del proyecto}

En esta sección se presentan la planificación docente de la asignatura, los problemas encontrados y las perspectivas futuras.

\subsection{Planificación de la propuesta docente}

La asignatura "G661 - Introducción a las Redes de Computadores" tiene 62 alumnos matriculados, descontando Erasmus. De acuerdo a los criterios establecidos, las sesiones de problemas en el aula asociadas a cada una de las sesiones de auto-aprendizaje se dividen en dos grupos diferentes. El horario de la titulación asigna estos dos grupos en martes y jueves respectivamente, lo que ha limitado en algún caso las semanas en las que era posible realizar el desdoble, al ser alguno de los dos días festivos.

Se han seleccionado seis sesiones para el aprendizaje autónomo del alumno. De estas seis sesiones, una no emplea vídeos (únicamente un texto escrito), pero otra emplea dos vídeos diferentes, por lo que se han desarrollado un total de seis vídeos para seis sesiones. Estas sesiones se han realizado durante Octubre (2 sesiones), Noviembre (2 sesiones), Diciembre (1 sesión) y Enero (1 sesión). Cada uno de los seis vídeos tiene asociado un test de autoevaluación asociado en el curso de Moodle de la asignatura. 
Aprendizaje autónomo del estudiante apoyado en recursos audiovisuales en el contexto de un Grado de Ingeniería Informática: experiencias con metodologías de enseñanza activas

Como métricas de seguimiento del proyecto, se han identificado los siguientes aspectos:

- Seguimiento de los recursos: proporción de alumnos que realizan las actividades de auto-aprendizaje, de acuerdo a las páginas de monitorización de los vídeos y los tests de autoevaluación.

- Encuesta de seguimiento para cuantificar la satisfacción del alumno.

- Resultados académicos de la asignatura: tasas de aprobado y porcentaje de alumnos presentados.

El único presupuesto disponible para la realización del proyecto está asignado a la difusión de resultados. Por este motivo, toda la realización de materiales audiovisuales se ha realizado por el equipo de trabajo, y las herramientas empleadas han sido libres y/o gratuitas.

No se contempla dentro del proyecto la modificación de los mecanismos de evaluación empleados en la asignatura. Indirectamente, esto permite la comparación de los resultados académicos del curso en que se ha desarrollado el proyecto frente a cursos anteriores.

\subsection{Herramientas de edición de vídeo y audio seleccionadas}

En primer lugar, se seleccionaron las herramientas técnicas apropiadas. Para el proceso de grabación y edición de vídeo y audio es necesario contar con unos conocimientos mínimos, si bien existen manuales paso por paso disponibles en la web.

Tras probar diferentes herramientas, el conjunto de herramientas empleado finalmente ha sido el siguiente:

- Captura de pantalla: OBS Studio 0.16.2. (Open Broadcaster Software 2017).

- Edición de vídeo: Avidemux 2.6.14 (Avidemux 2017) para el recortado y concatenado sin recodificación de pistas de vídeo o audio; ShotCut (Meltytech 2017) para la edición no lineal y recodificación de vídeo.

- Edición de audio: Audacity 2.1.2 (Audacity 2017) para desarrollar efectos de audio en cortinillas de cierre y reducción de ruido.

La sección 2.4 detalla algunos problemas sufridos en el proceso de elección de herramientas.

\subsection{Materiales desarrollados}

Se han desarrollado seis vídeos, seis tests de autoevaluación y seis sesiones de problemas en el aula.

Los vídeos desarrollados se han capturado a partir de la pantalla del ordenador personal, en múltiples cortes. Para ello, se ha empleado la herramienta OBS Studio seleccionando el codec H.264 con una resolución de 1366x768 (suficiente para una visualización clara por parte del alumno). El audio del profesor se ha capturado a partir de un micrófono integrado en el monitor. Las diferentes pistas capturadas se 


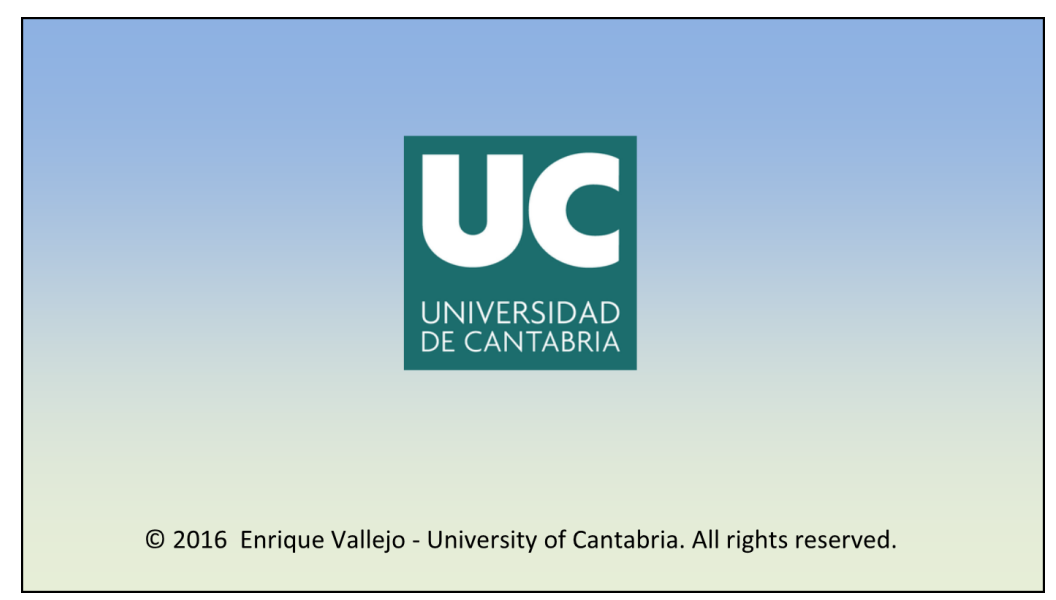

Fig. 1: Sencilla cortinilla empleada para remarcar el final de los vídeos.

han recortado (al comienzo y final de la misma) ajustando a un keyframe para evitar la recodificación de toda la secuencia empleando Avidemux, y también se han concatenado con esta herramienta. La pista de audio se ha editado con Audacity para reducir el ruido de fondo. Por otra parte, se ha desarrollado una cortinilla que indica el final del vídeo basado en una imagen estática con un fundido realizado con ShotCut, combinado con unos todos desarrollados con Audacity. La imagen empleada en la cortinilla final se muestra en la figura 1. Por desgracia, por una incompatibilidad entre la codificación de las secuencias, el concatenado de esta cortinilla ha requerido la recodificación de la secuencia completa, lo que podía implicar un proceso de unos 45 minutos por vídeo; este tiempo, en cualquier caso, depende de las prestaciones del equipo en que se realiza la recodificación.

Debido a su tamaño, los vídeos no pueden hospedarse en el servidor de Moodle de la Universidad. Consultados los técnicos del Centro de Formación en Nuevas Tecnologías de la Universidad de Cantabria (CeFoNT), estos vídeos se alojaron en YouTube, y se enlazaron en el curso de Moodle de la asignatura, incrustados en un elemento del tipo "Página" que permite presentar un código HTML.

La Figura 2 muestra la página de gestión de YouTube con los 6 vídeos publicados. Esta página incluye también una estadística básica del número de visionados de cada uno de los vídeos, que no implica la visualización del vídeo completo.

Los materiales desarrollados se han basado en los contenidos tradicionalmente empleados en la asignatura, si bien se ha intentado que el vídeo sea lo más demostrativo posible, y no una simple (y desmotivadora) sucesión de transparencias. En este sentido, se han grabado varias demos (por ejemplo, para temas de codificación de audio y vídeo para su transmisión por la red) y se han añadido múltiples animaciones en las transparencias empleadas. La duración de cada vídeo se comprende entre 25 y 32 minutos, significativamente inferior a una clase teórica.

Asociado a cada uno de los vídeos, se ha desarrollado un test de autoevaluación en Moodle. Aunque los alumnos reciben una nota tras finalizarlo, este test no tiene peso en la calificación de la asignatura, y está destinado únicamente al auto-aprendizaje 


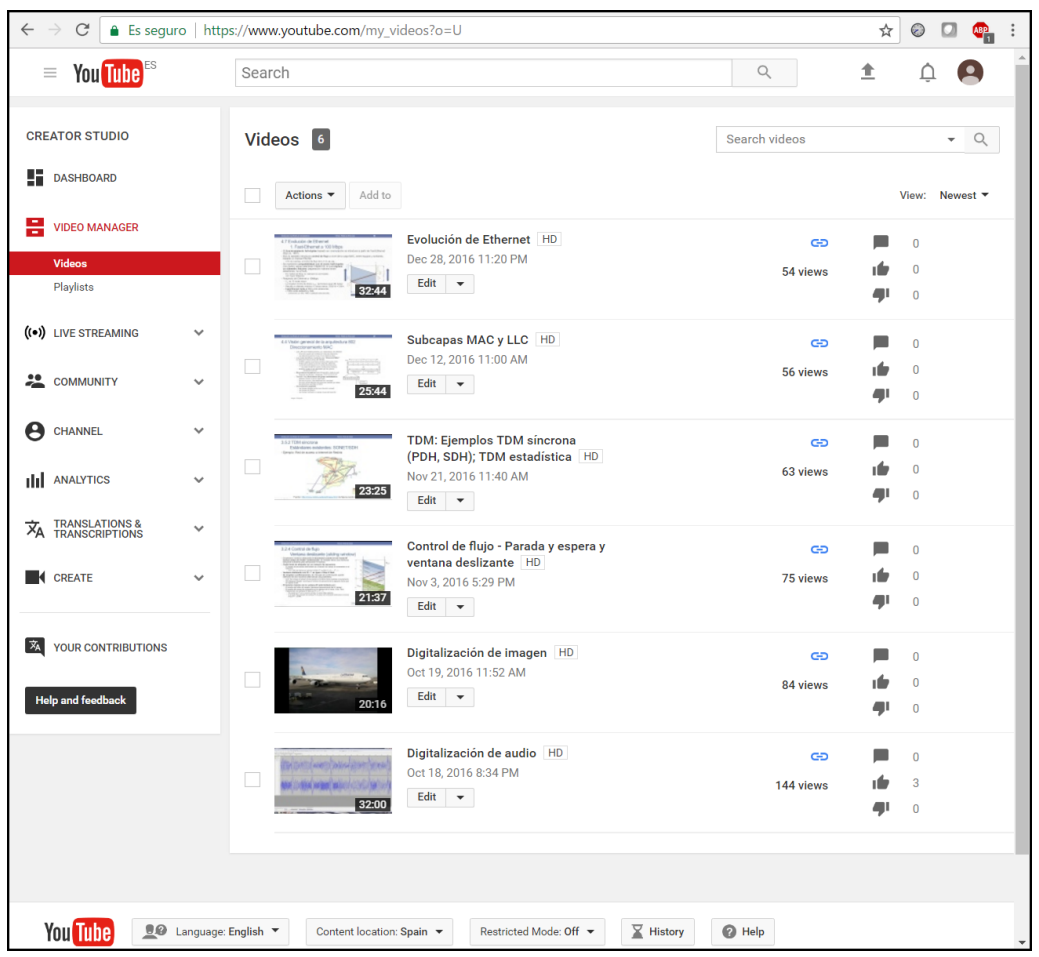

Fig. 2: Captura de la interfaz de gestión de los vídeos subidos en YouTube.

por parte del alumno. Se han desarrollado por tanto seis tests diferentes, con entre 3 y 6 preguntas por test. Las preguntas comprenden preguntas de diferentes tipos, incluyendo respuesta múltiple con una o varias respuestas posibles, respuesta corta, relacional y preguntas calculadas. La Figura 4 muestra una captura de uno de los tests de autoevaluación desarrollados.

Para las sesiones presenciales en el aula, se ha asignado una temática diferente a cada uno de los dos grupos de alumnos. En un grupo, se ha planteado un estudio de la tecnología HFC (Hybrid Fiber-Coaxial), la tecnología empleada para prestar servicios de acceso a Internet por operadores de cable. En el otro grupo, se ha centrado el estudio en la tecnología de acceso a Internet por fibra óptica, basada en el estándar más extendido en España GPON, G.984. En ambos casos, las actividades realizadas comprenden los diferentes aspectos que se estudian en la asignatura, como puede ser la codificación de señales digitales, multiplexación, cálculo de la velocidad de transmisión, cálculo de overheads, mecanismos de control de flujo, etc.

Aprovechando el proceso, se han desarrollado también tests de autoevaluación para el resto de contenidos de la asignatura, pensados como apoyo al repaso de los contenidos impartidos en las sesiones presenciales. La Figura 3 muestra el total de tests de autoevaluación disponibles en Moodle para los alumnos, junto con el número de intentos realizados en cada uno de ellos una vez finalizado el periodo docente. La figura 4 muestra los contenidos de parte de uno de los tests. 


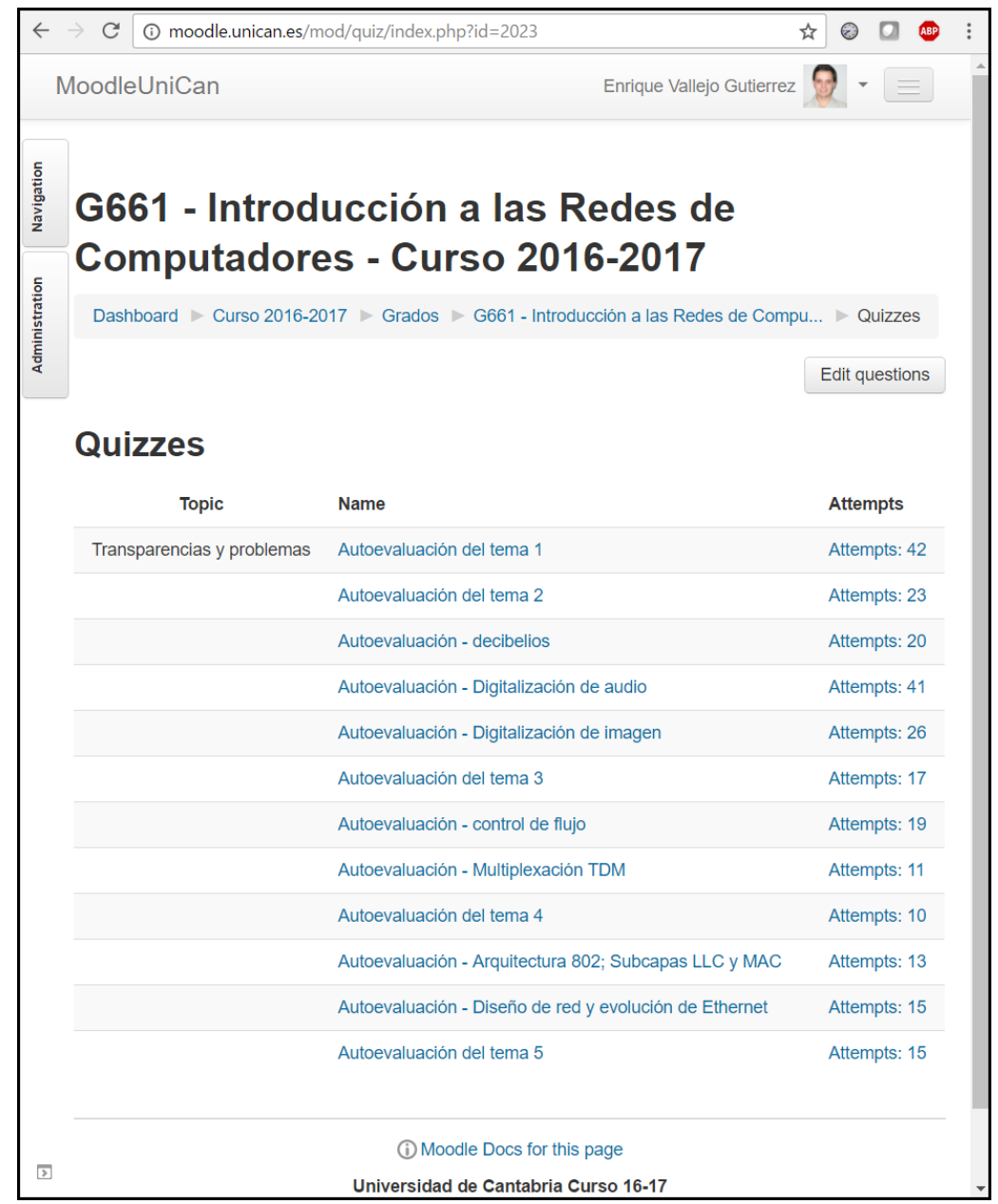

Fig. 3: Captura de pantalla de los tests desarrollados en Moodle.

Las sesiones de prácticas en el aula desarrolladas en el contexto de este proyecto han seguido un estudio de un caso de una tecnología concreta. A un grupo se le han desarrollado una serie de problemas sobre los accesos a Internet por fibra óptica mediante tecnología GPON, y al otro grupo un conjunto similar de problemas sobre el acceso mediante redes híbridas de fibra-coaxial (DOCSIS). El motivo para elegir estas temáticas (además de la resolución de problemas más específicos de la asignatura) es doble: Por una parte, estas dos son las tecnologías de acceso a Internet de alta velocidad más extendidas, y se han desarrollado los problemas para que resulten llamativos al alumno. Por otra parte, al presentar un sistema completo se le presenta al alumno un sistema muy complejo en el que se estudian los diferentes elementos (aspectos de transmisión de la señal, codificación y modulación, multiplexación, control de acceso al medio, etcétera), lo que hace que afronte un problema complejo con múltiples variables, similar al considerado en los exámenes de la asignatura. 


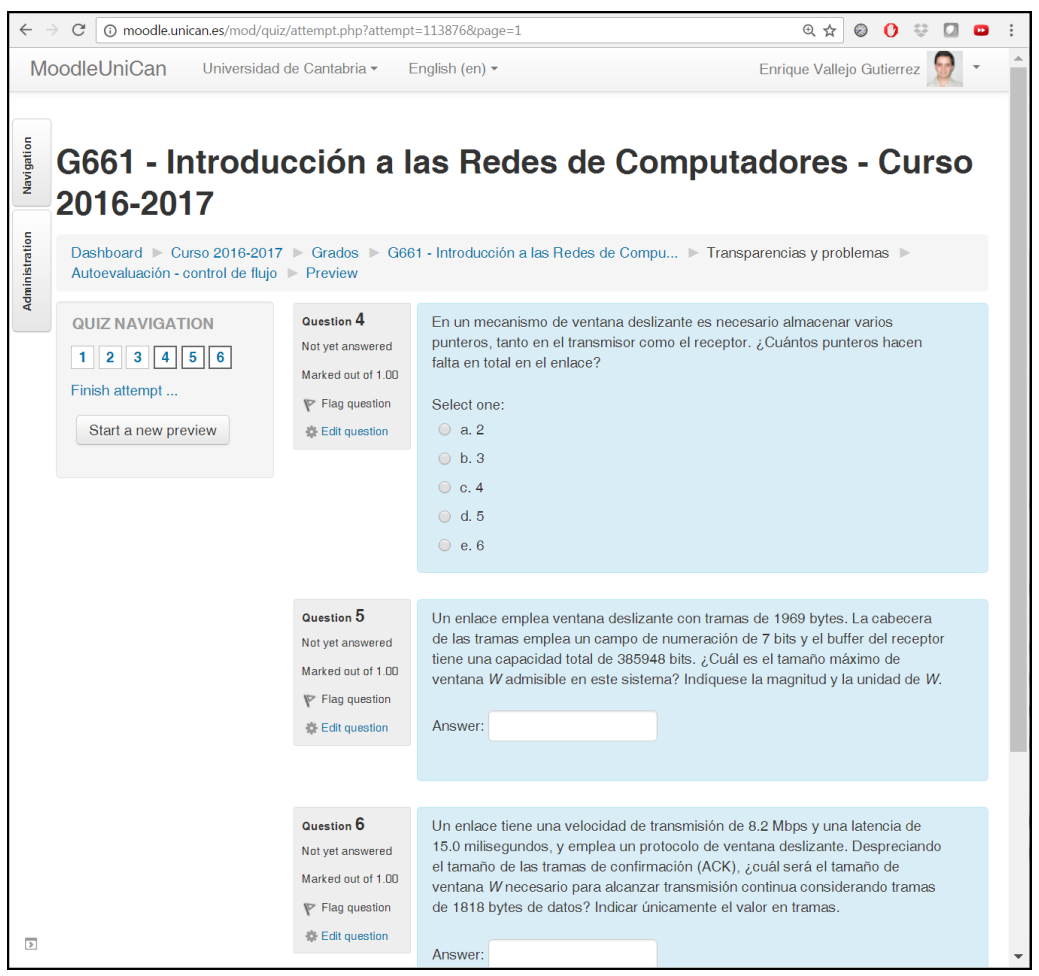

Fig. 4: Captura de uno de los tests de Moodle empleados como apoyo de la sesión de auto-aprendizaje.

\subsection{Dificultades y problemas técnicos encontrados}

En esta sesión se comentan algunas dificultades encontradas durante las primeras fases del desarrollo del contenidos para el proyecto.

Por una parte, la selección de las herramientas de captura y edición de vídeo apropiadas ha sido más complejo de lo pensado inicialmente. La solicitud inicial del proyecto contemplaba una partida económica destinada a la compra de un software comercial apropiado y suficientemente potente y estable, pero al no asignarse, se han analizado diferentes herramientas gratuitas. Varias herramientas probadas durante la fase inicial resultaron inapropiadas. En algún caso, esto se debió a estar limitadas en sus capacidades; por ejemplo, Microsoft Expression Encoder 4 (que es la herramienta empleada en alguno de los cursos de formación de la Universidad de Cantabria) en su versión gratuita no permite la codificación en H.264 (códec apropiado para la distribución de vídeo en alta definición). En algún otro caso, se probaron aplicaciones que sí disponían de suficientes prestaciones técnicas, pero que no eran lo suficientemente estables y fallaban durante la ejecución; por ejemplo, el editor no lineal OpenShot servía para el propósito buscado, pero fallaba (perdiendo todo el trabajo realizado) en ciertas operaciones, como la previsualización de un vídeo cargado. En concreto, con esta herramienta se probaron diferentes versiones, tanto estables como versiones de desarrollo, sin encontrar ninguna lo suficientemente estable, lo que resultó en una pérdida de tiempo considerable. 
Por otra parte, también se consideró en el presupuesto inicial la compra de un monitor, si bien este tipo de gastos no se consideraba dentro del proyecto. El uso de un único monitor ha resultado problemático en varios casos. En concreto, en varias ocasiones el programa de captura ha fallado mientras se ejecutaba, pero al disponer a pantalla completa de los materiales de la asignatura (que se pretendían capturar) no se ha apreciado el fallo. Este tipo de problemas se han resuelto al encontrar un monitor viejo que no tenía uso, y emplearlo de apoyo durante la captura.

Finalmente, se ha encontrado que es necesario un esfuerzo muy significativo en la preparación de los materiales. Aunque ya se disponía de transparencias de la asignatura del curso previo, éstas estaban orientadas a apoyar una clase presencial muy basada en las explicaciones en la pizarra. Al no disponer de la misma, ha sido necesario adaptar significativamente algunos aspectos, aumentando la cantidad de animaciones empleadas y el uso de contenidos adicionales (capturas de "demos"). De forma similar, también ha sido necesario preparar de forma muy clara un guion muy claro para cada uno de los elementos a grabar, ya que la improvisación que puede emplearse en una clase presencial interactiva no resulta viable en una grabación, y una pequeña duda o vuelta atrás resulta mucho más indeseable.

\section{Respuesta del alumnado e impacto del programa}

En esta sección se comenta la realimentación obtenida de los alumnos sobre el programa realizado. Para ello, se comentan primero las impresiones proporcionadas por los alumnos en conversaciones informales. Después, se analizan las métricas de seguimiento proporcionadas por las herramientas informáticas. Finalmente, se analizan los resultados académicos obtenidos en la única convocatoria de la asignatura hasta la fecha.

\subsection{Impresiones informales de los alumnos}

En conversaciones informales, los alumnos indican que les parecen muy interesantes los vídeos empleados, tanto en los contenidos como el formato, y que prefieren este tipo de enfoque docente frente a las clases magistrales tradicionales. Algún alumno sugería partirlos en trozos más pequeños (de los 20-30 minutos actuales a dos vídeos de 10-15 minutos); aunque esto no parece algo viable a priori (ya que no parece suficiente para cubrir un concepto relativamente complejo) se puede analizar para cursos posteriores.

\subsection{Seguimiento de los vídeos y tests}

Sin embargo, las herramientas de monitorización indican que el seguimiento es inferior a lo deseado, y decreciente según avanza el tiempo. En la plataforma de YouTube se proporcionan estadísticas sobre el visionado de los vídeos. En dichas estadísticas se observa que la cantidad de visionados por vídeo es inferior al número de alumnos, y que desciende según avanza la asignatura (de casi un $90 \%$ en los primeros vídeos a menos de un $50 \%$ en los últimos). Aunque estas estadísticas son coherentes con la asistencia a clase habitual en el plan de estudios (y con su evolución decreciente según avanza el cuatrimestre), sería deseable obtener valores más elevados. La figura 5 muestra estadísticas del primero de los vídeos durante las primeras dos semanas y media desde su publicación. En concreto, se observa cómo el vídeo se ha visto varias veces, si bien 
Aprendizaje autónomo del estudiante apoyado en recursos audiovisuales en el contexto de un Grado de Ingeniería Informática: experiencias con metodologías de enseñanza activas

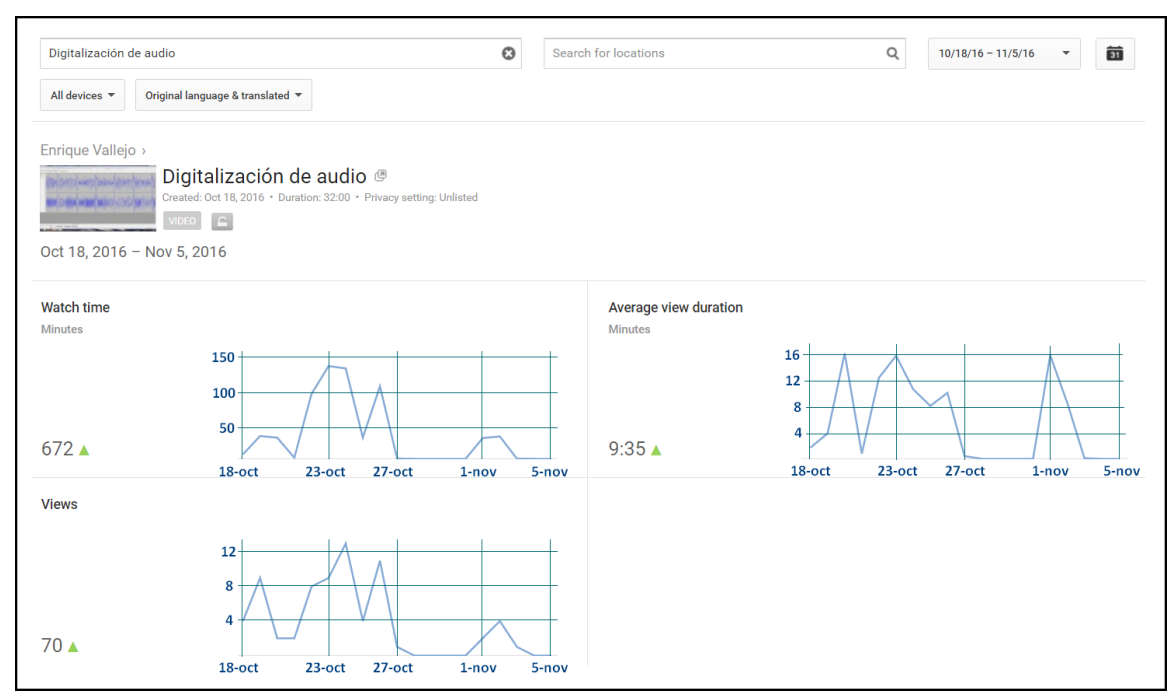

Fig. 5: Captura de estadísticas de visionado del vídeo relativo a codificación de audio, proporcionadas por la herramienta de análisis de YouTube. Imagen editada para incluir los ejes de referencias para facilitar su análisis.

la duración promedio de cada visionado no alcanza la tercera parte de la duración del vídeo. En general, el tiempo de visionado promedio de cada vídeo ha cubierto únicamente entre un $29 \%$ y un $42 \%$ de la duración del mismo. Aun descontando una estimación de los visionados de prueba realizados durante la preparación del vídeo, esto se traduce en una penetración baja en el público objetivo.

Este análisis lo corroboran los tests de autoevaluación de Moodle. La Figura 3 contiene el número de intentos realizados en cada uno de los tests. Considerando que el número de alumnos es de 62, en solo dos casos se ha llegado a un $50 \%$ de intentos del test de autoaprendizaje respecto al total de alumnos en la asignatura. En el peor caso, llega a haber únicamente 13 intentos $^{1}$, en torno al $10 \%$. Preguntados al efecto, algunos alumnos sugieren la aplicación de bonus en la calificación por el hecho de realizar estos tests como herramienta de motivación; es decir, entienden los tests como una carga y no como una ayuda a su proceso de aprendizaje.

\footnotetext{
${ }^{1}$ En algún caso hay valores inferiores, pero no se corresponden a la autoevaluación asociada a un vídeo, sino a un tema completo.
} 


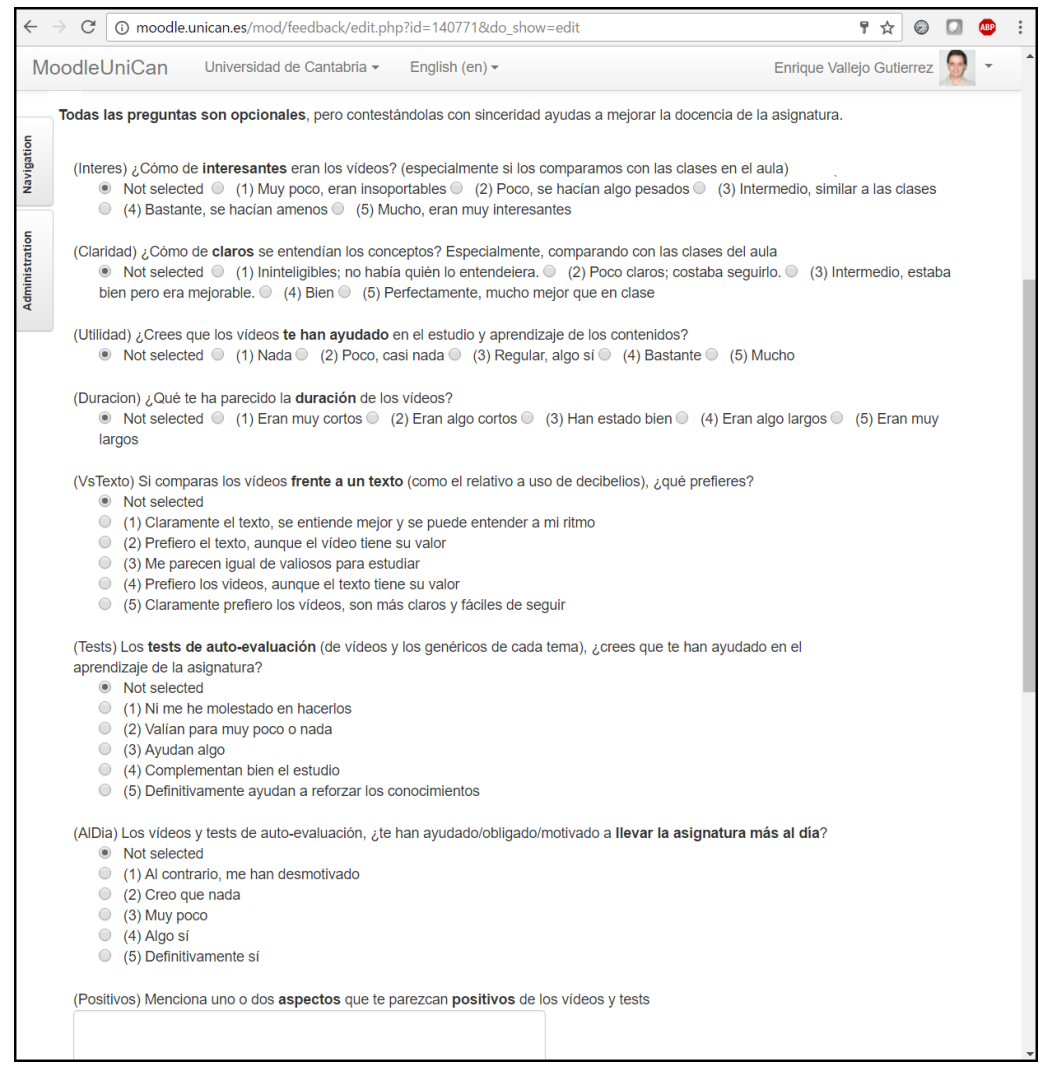

Fig. 6: Captura de las primeras 8 preguntas de la encuesta de satisfacción al alumnado.

\subsection{Encuesta de satisfacción}

Se ha realizado a los alumnos una encuesta de satisfacción sobre el programa piloto. La encuesta se realizaba a través de la página de Moodle de la asignatura, y contenía un total de 9 preguntas de selección más tres preguntas abiertas: aspectos positivos, aspectos negativos y sugerencias. La figura 6 muestra las primeras preguntas de la encuesta; las preguntas de selección restantes preguntan por el número de sesiones y la satisfacción general.

La encuesta se puso a disposición de los alumnos en la última quincena de curso y se dejó abierta, sin fecha límite de finalización. Se animó insistentemente a los alumnos a participar, pero no se les incentivó de ninguna manera. En total, se recogieron 24 respuestas de los alumnos. El significado de cada una de las puntuaciones puede encontrarse en la figura 6 para las primeras 8 preguntas, y se indica para las siguientes. La calificación media de las preguntas de selección se muestra a continuación:

- ¿Cómo de interesantes eran los vídeos? Calificación promedio: 3.83

- ¿Cómo de claros se entendían los conceptos? Calificación promedio: 4.00 


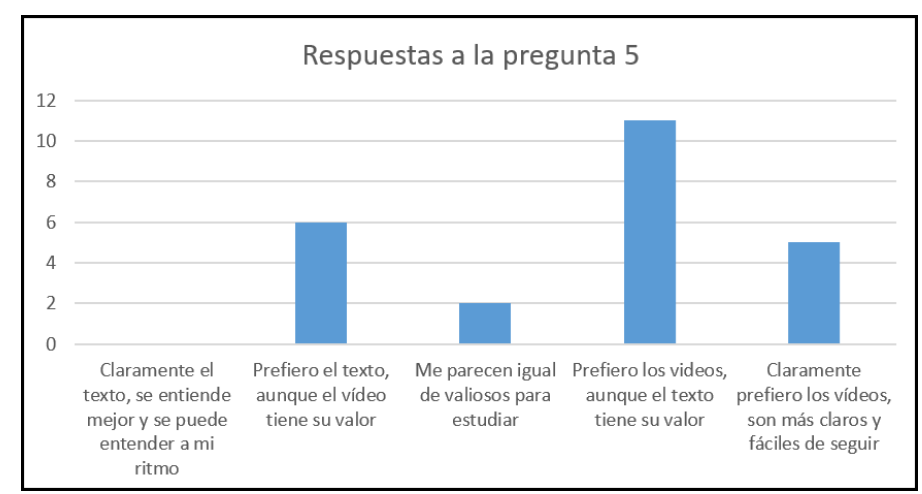

Fig. 7: Respuestas a la pregunta 5: Si comparas los vídeos frente a un texto (como el relativo a uso de decibelios), ¿qué prefieres?

- ¿Crees que los vídeos te han ayudado en el estudio y aprendizaje de los contenidos? Calificación promedio: 3.92

- ¿Qué te ha parecido la duración de los vídeos? Calificación promedio: 3.75

- Si comparas los vídeos frente a un texto (como el relativo a uso de decibelios), ¿qué prefieres? Calificación promedio: 3.63

- Los tests de auto-evaluación (de vídeos y los genéricos de cada tema), żcrees que te han ayudado en el aprendizaje de la asignatura? Calificación promedio: 3.04

- Los vídeos y tests de auto-evaluación, ¿te han ayudado/obligado/motivado a llevar la asignatura más al día? Calificación promedio: 3.25

- Respecto al número de sesiones con vídeos de auto-aprendizaje, ¿cuántas harías? (1 - Muchas menos o ninguna; 5 - Casi todas las clases basadas en vídeos) Calificación promedio: 3.79

- ¿Cuál es tu satisfacción general con este programa piloto? (1 - No me ha gustado nada; decepcionante; 5 - Me ha gustado mucho) Calificación promedio: 3.96.

En todas las preguntas, las respuestas han seguido una distribución unimodal, excepto en las relativas a la preferencia por un texto o un vídeo (marcada como VsTexto en la figura 6), y en los tests de autoevaluación. En el segundo caso, había un pequeño pico de tres alumnos que indicaban que no se habían molestado en responder a los tests. En el primer caso, la figura 7 muestra la distribución de respuestas, en donde valores a la izquierda indican la preferencia por un texto y valores a la derecha por un vídeo.

En cuanto a los comentarios recibidos, los principales aspectos valorados positivamente han sido la posibilidad de repetir el vídeo si algo no se entiende, la claridad en la explicación (enlazado con el hecho de que sea audiovisual) y la flexibilidad horaria. Los principales aspectos negativos se han centrado en la duración excesiva de los vídeos, la dificultad de los tests y el tipo de pregunta que difiere del examen final, y la cantidad 
de contenidos que perciben los alumnos. En cuanto a sugerencias, las respuestas fueron bastante variadas, desde insistir en acortar la duración, solicitar calificación extra por completar los tests de auto-evaluación y realizar la mayor cantidad de problemas en el aula posibles. En general, muchos comentarios recibidos siguen la misma línea que los observados en trabajos previos (Ronchetti 2010).

\subsection{Resultados académicos}

En el momento de escribir este documento solo se ha realizado una de las dos convocatorias anuales de la asignatura. El número de alumnos presentados fue de 43 , un $69,4 \%$ de los alumnos matriculados. Comparativamente, este porcentaje en una única convocatoria en los tres cursos anteriores había variado en el rango $65 \%-82 \%$, por lo que no puede determinarse que el cambio metodológico haya influido en el porcentaje de alumnos presentado.

Respecto al número de alumnos que aprueban la asignatura, la tasa de aprobados frente a matriculados en una convocatoria ha sido de un $41,9 \%$. En los tres cursos anteriores, en la convocatoria de Febrero esta tasa se ha movido en el rango 23,4\%-38,9\%, por lo que el resultado académico sí que puede considerarse claramente superior al de cursos previos.

\subsection{Análisis subjetivo}

En esta sección se presenta una valoración de la experiencia observada por el profesor de la asignatura. Esta valoración es subjetiva, y se basa únicamente en una impresión personal y un análisis propio de los resultados.

En primer lugar, se ha recibido una respuesta muy positiva de la gran mayoría de los alumnos, tanto en comentarios individuales como en la encuesta de valoración del proyecto o de la asignatura; algunos alumnos han aprovechado las mismas para mostrar su aprecio, agradeciendo el trabajo y animando a seguir por el mismo camino.

Sorprendentemente, los tests de autoevaluación no han sido muy seguidos por los alumnos. Estos tests se han desarrollado como una ayuda al estudio, pero los alumnos lo han percibido como una tarea (carga) adicional, y no la han realizado ya que no tenía impacto directo en la nota final. Aparentemente, no aprecian el valor de los mismos como herramienta de aprendizaje. La dificultad de los tests era deliberadamente elevada, para exigir bastante a los alumnos tras cada visualización de un contenido, pero en algunos casos esto les echaba para atrás.

Resulta muy llamativa la respuesta ante la preferencia por medios escritos o audiovisuales. En opinión del profesorado, siempre resulta más claro y práctico un buen libro o texto escrito que un vídeo, tanto por la facilidad para hojearlo o leerlo en diagonal, como por la facilidad de seguirlo con el detenimiento necesario. En este sentido, la respuesta de los alumnos mostrada en la figura 7 es variada, existiendo dos tendencias pero una mayoría que se decanta por preferir el uso de materiales audiovisuales. Probablemente esto no es más que una muestra de la evolución de la sociedad, a la que debe adaptarse el proceso de enseñanza y aprendizaje.

El esfuerzo destinado a la preparación de contenidos ha sido muy significativo. Por cada vídeo de unos 20-30 minutos ha resultado necesario invertir varias horas de trabajo, 
si se tiene en cuenta la preparación de software, desarrollo de contenidos, múltiples pruebas, grabación, edición, subida a Internet, etc. El esfuerzo es muy elevado y no resulta fácilmente visible para los alumnos. Por otra parte, las clases prácticas en el aula no han seguido una metodología pura de aprendizaje basado en problemas, dada la poca proactividad de los alumnos a colaborar en grupos siguiendo diferentes roles. Este aspecto se debe trabajar más en ocasiones posteriores.

Resulta ligeramente decepcionante que la tasa de alumnos presentados se haya mantenido en el mismo rango que en años anteriores, si bien esto se compensa con la mayor tasa de aprobados dentro de los alumnos que sí se han presentado. En este sentido, el proyecto puede considerarse generalmente positivo.

\section{Conclusiones y perspectivas de futuro}

De acuerdo al análisis presentado en las secciones previas, puede considerarse que el proyecto se ha desarrollado con relativo éxito.

Se ha evaluado el impacto del proyecto sobre la motivación, seguimiento y resultados del alumnado mediante varias actuaciones: una encuesta informal en el aula, la monitorización del seguimiento de las herramientas informáticas, el uso de una herramienta de encuesta formal online, y el análisis de resultados académicos frente a los obtenidos en años previos. Las respuestas informales recibidas en el aula sugieren que los alumnos prefieren este tipo de docencia frente a las clases seminales tradicionales. Sin embargo, las herramientas de monitorización de los vídeos y tests de autoevaluación sugieren que el seguimiento es bajo, mucho más de lo esperado.

Los resultados académicos son positivos, con una tasa de aprobados más elevada que en convocatorias equivalentes de cursos previos, pero con una tasa de alumnos presentados más baja. En este sentido, resulta llamativo que los alumnos han valorado positivamente el programa pero no está claro si ha influido positivamente en su motivación a no abandonar la asignatura. Eso sí, el hecho de que la tasa de alumnos aprobados frente a presentados sea significativamente más elevada es una muestra clara del resultado positivo.

Para cursos posteriores se planteará mantener el programa seguido, y si es posible aumentarlo. Sin embargo, el esfuerzo requerido por parte del profesor es significativo y no se ve recompensando claramente. Sería interesante estudiar la posibilidad de una valoración expresa de este tipo de actividades por parte de los organismos académicos.

\section{Referencias bibliográficas}

Audacity (2017). Audacity website. URL: http://www . audacityteam.org/ (visitado 05-10-2016).

Avidemux (2017). Avidemux website. URL: http://fixounet.free.fr/avidemux/ (visitado 05-10-2016).

Lage Maureen J., Glenn J. Platt y Michael Treglia (2000). "Inverting the Classroom: A Gateway to Creating an Inclusive Learning Environment". En: The Journal of Economic Education 31.1, págs. 30-43. DOI: 10.2307/1183338. 
Meltytech, LLC (2017). ShotCut website. URL: https: //www. shotcut.org/ (visitado 05-10-2016).

Open Broadcaster Software (2017). OBS Studio. URL: https ://obsproject.com/ (visitado 05-10-2016).

Ronchetti, Marco (2010). "Using video lectures to make teaching more interactive". En: International Journal of Emerging Technologies in Learning (iJET) 5.2, págs. 45-48. ISSN: 1863-0383.

Santiago, Raúl y Alicia Díez (2016). The Flipped Classroom. URL: http: / / www . theflippedclassroom.es/ (visitado 10-09-2016).

Sparks, Sarah D. (2011). Lectures Are Homework in Schools Following Khan Academy Lead. URL: http://www . edweek .org/ew/articles/2011/09/28/05khan_ep. h31.html (visitado 15-03-2017).

Wood, Diana F (2003). "Problem based learning". En: BMJ 326.7384, págs. 328-330. ISSN: 0959-8138. DOI: 10 .1136/bmj . 326.7384 .328. eprint: http://www . bmj . com/content/326/7384/328.full.pdf. 